\title{
Plasmon Driven Nanoparticle Movement in the Electron Beam
}

\author{
A. Reyes-Coronado ${ }^{1}$, J. Aizpurua ${ }^{1,2}$, P.M. Echenique ${ }^{1,2}$, R.G. Barrera ${ }^{3}$, P.E. Batson ${ }^{4,5}$ \\ ${ }^{1}$ Donostia International Physics Center, Spain, ${ }^{2}$ Centro de Física de Materiales CSIC- \\ UPV/EHU, Spain, ${ }^{3}$ Instituto de Física, Universidad Nacional Autónoma de México, Mexico, \\ ${ }^{4}$ IBM Thomas J. Watson Research Center, USA, ${ }^{5}$ Institute for Advanced Materials, Devices, \\ and Nanotechnology, Rutgers University, USA.
}

During initial work with aberration corrected STEM imaging, it became clear that observation of metallic nanoparticles smaller than about $5 \mathrm{~nm}$ in size results in copious particle movement [1], supporting earlier TEM observations of very small nanoparticles [2]. This behavior appears to be a result of two new types of behavior: 1) sub-Ångstrom STEM imaging injects enough energy into local structures that atomic bonds are destabilized, allowing easier atomic movement among alternative bonding sites; and 2) the resulting motion of sub-5 nm sized metallic clusters on carbon is non-random and apparently directed by forces induced by the passing STEM electron beam. This report summarizes results of theoretical work, aimed at understanding the motion of metallic clusters in the presence of the electron beam.

As has been recognized for many years in the context of EELS, when a fast electron passes a small object, it induces a dielectric response which produces electron energy loss [3]. It has also been recognized that this dielectric behavior is responsible for many types of attractive forces: VanderWaals dipole-dipole forces, London Dispersion fluctuation forces, Debye induced dipole forces, and Keesom forces between electrostatic charges. Extensive work on these phenomena for estimation of interfacial forces in ceramics from measurements of valence EELS has been discussed by French [4].

In this case, as summarized in Fig. 1, (on the right, below) nanometer-sized Au clusters readily move under the electron beam, often coalescing with neighboring clusters. Motion is non-random, most often directed towards a neighboring cluster after a latency period that depends on beam current density, and often resulting in violent coalescence with a few $\mathrm{Au}$ atoms left bonded to the carbon substrate. In Fig. 2, (on the left, below) an experimental EELS result using oxide-coated Al spheres is shown, reproduced from [5]. This experiment showed that, when the fast electron passes near the end of one of the spheres (position D in the Fig. 2), a coupling of the surface plasmons on two nearby spheres produces a surface plasmon resonance having bi-spherical symmetry and an energy of about $4 \mathrm{eV}$ - about half the normal $7 \mathrm{eV}$ energy for an oxide-coated $\mathrm{Al}$ sphere. Also in Fig. 2. we show a model structure used to calculate the mutual forces between the two metal spheres for this case. Finally we show the bi-spherical plasmon field intensity, and phase indicated by the plus/minus signs. The important point to realize is that although the surface plasmon field is oscillating rapidly (of order $10^{14} \mathrm{~Hz}$ ), the field is always anti-symmetric between the two spheres, resulting in an attractive force, for the indicated fast electron impact position.

Similar calculations for single spheres at moderate distances shows a weakly attractive force 
directed towards the electron beam, in agreement with earlier work [6]. In this case, the presence of the second sphere reverses the direction of the force and increases its magnitude by $10 \mathrm{x}$ to $5-10$ picoNewtons. This is a moderate force, but less than a typical force applied using optical tweezers - of order $70 \mathrm{pN}$ for about $5 \mathrm{MW} / \mathrm{cm}^{2}$ light intensity [7]. Once the particle under the beam becomes loose from the carbon substrate, we expect it to move quite fast, $\simeq 1200 \mathrm{~nm} / \mathrm{sec}$.

If the fast electron does not pass near the end of one of the spheres - for instance if it passes near $\mathrm{C}$ in Fig. 2. - then coupling to the bi-spherical mode cannot happen, and the attractive force should disappear. We will show an example of this behavior, thus verifying that this physical picture is likely correct.

[1] P. E. Batson, Micros. Microanal., 14 (2008) $89-97$.

[2] L. D. Marks, Rep. Prog. Phys., 57 (1994) $603-649$.

[3] R. H. Ritchie, Phys. Rev., 106 (1957) 874-881.

[4] R. H. French, J. Amer. Ceram. Soc., 83 (2000) 2117-2146.

[5] P. E. Batson, Surface Science, 156 (1985) $720-734$.

[6] F. J. García de Abajo, Phys. Rev. B, 70 (2004) 115422.

[7] A. J. Hallock, P. L. Redmond, and L. E. Brus, Proc. Nat. Acad. Sci., 102 (2005) 1280-1284.
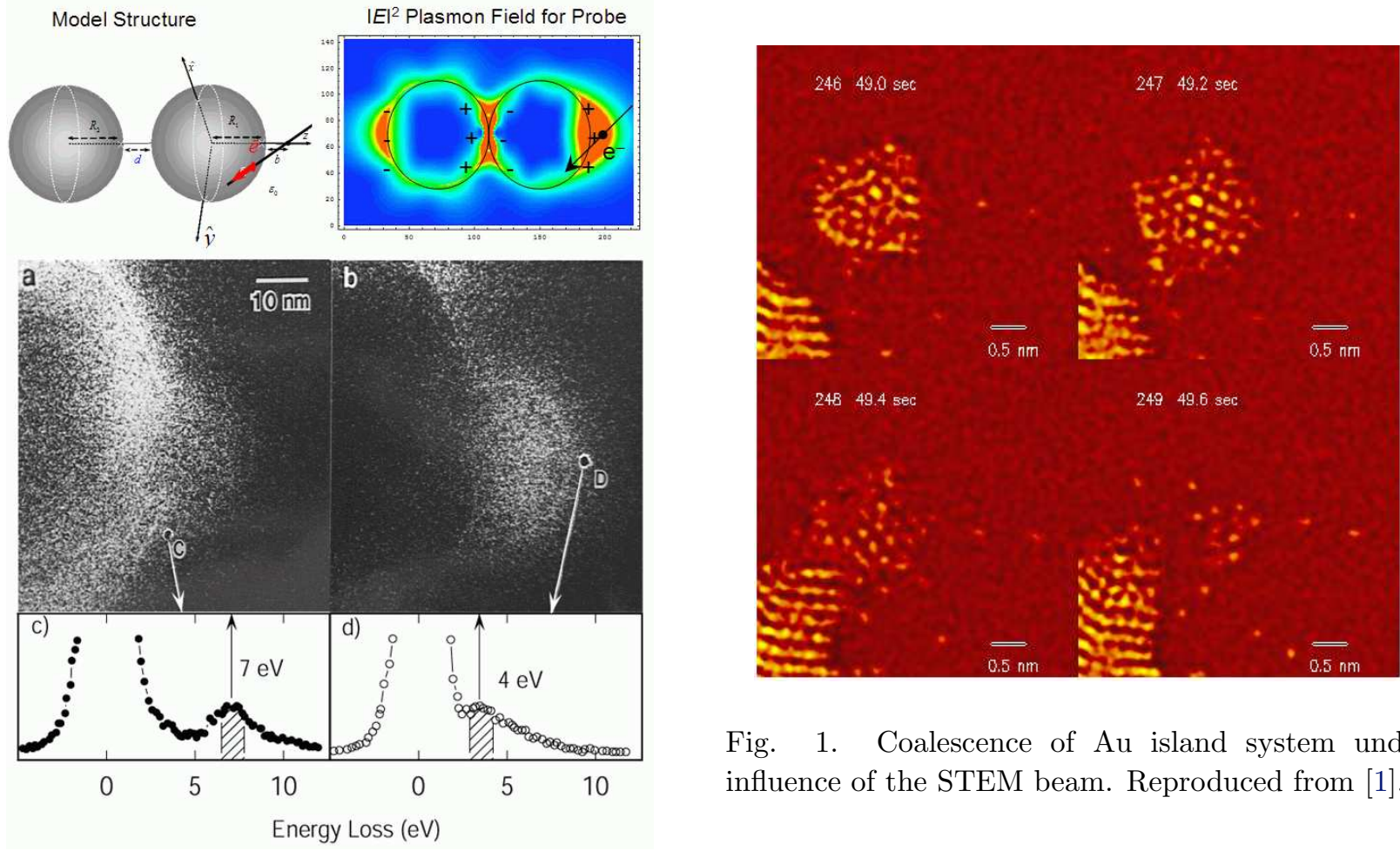

Fig. 1. Coalescence of $\mathrm{Au}$ island system under influence of the STEM beam. Reproduced from [1].

FIG. 2. (bottom) Bispherical surface plasmon EELS experiment (reproduced from [5]) showing EELS spectra for two probe positions, and the scattering intensity maps generated using the two observed peaks in the EELS spectra. (Top Left) Model geometry for calculations of electric field and resulting forces for a passing fast electron. (Top Right) The resulting magnitude of the induced electric field between the spheres. The phase of the induced charges is indicated by the signs. 